\title{
DEVELOPMENT OF METHODS FOR SUPPLY MANAGEMENT IN TRANSPORTATION NETWORKS UNDER CONDITIONS OF UNCERTAINTY OF TRANSPORTATION COST VALUES
}

\author{
Lev Raskin \\ Department of Distributed Information Systems and Cloud Technologies ${ }^{1}$ \\ Oksana Sira \\ Doctor of Technical Sciences, Professor \\ Department of Distributed Information Systems and Cloud Technologies ${ }^{1}$ \\ topology@ukr.net \\ Yurii Parfeniuk \\ Department of Distributed Information Systems and Cloud Technologies ${ }^{I}$ \\ parfuriy.l@gmail.com \\ Kseniia Bazilevych \\ Department of Mathematical Modeling and Artificial Intelligence \\ National Aerospace University «Kharkiv Aviation Institute» \\ 17 Chkalova str., Kharkiv, Ukraine, 61070 \\ k.bazilevych@khai.edu \\ ${ }^{I}$ National Technical University «Kharkiv Polytechnic Institute» \\ 2 Kyrpychova str., Kharkiv, Ukraine, 61002
}

\begin{abstract}
The problem of transport management in a distributed logistics system «suppliers - consumers» is considered. Under the assumption of a random nature of transportation costs, an exact algorithm for solving this problem by a probabilistic criterion has been developed. This algorithm is implemented by an iterative procedure for sequential improvement of the transportation plan. The rate of convergence of a computational procedure to an exact solution depends significantly on the dimension of the problem and is unacceptably low in real problems. In this regard, an alternative method is proposed, based on reducing the original problem to solving a nontrivial problem of fractional-nonlinear programming. A method for solving this problem has been developed and substantiated. The corresponding computational algorithm reduces the fractional-nonlinear model to the quadratic one. The resulting problem is solved by known methods. Further, the original problem is supplemented by considering a situation that is important for practice, when in the conditions of a small sample of initial data there is no possibility of obtaining adequate analytical descriptions for the distribution densities of the random costs of transportation. In this case, the available volume of statistical material is sufficient only to estimate the first two moments of unknown distribution densities. For this marginal case, a minimax method for finding the transportation plan is proposed. The first step is to solve the problem of determining the worst distribution density with the given values of the first two moments. In the second step, the transportation plan is found, which is the best in this most unfavorable situation, when the distribution densities of the random cost of transportation are the worst. To find such densities, let's use the modern mathematical apparatus of continuous linear programming.
\end{abstract}

Keywords: transport linear programming problem, random transportation costs, exact solution algorithm, worst distribution density, minimax solution.

DOI: $10.21303 / 2461-4262.2021 .001691$

\section{Introduction}

In the system of management tasks for the multifunctional logistics complex «production delivery - consumption», transport tasks take the central place.

The transport linear programming problem in the traditional setting is formulated as follows [1-3]. There are $m$ suppliers of a homogeneous product and $\mathrm{n}$ consumers of this product. It is known the vector $A=\left(a_{1} a_{2} \ldots a_{i} \ldots a_{m}\right)$, the components of which fix the capabilities of suppliers, 
the vector $B=\left(b_{1} b_{2} \ldots b_{j} \ldots b_{n}\right)$, the components of which specify the demand of consumers, and also the matrix $C=\left(c_{i j}\right)$, which determines the cost of delivery of a product unit from suppliers to consumers. It is necessary to find a nonnegative matrix $X=\left(x_{i j}\right)$ that defines a plan for transporting a product from suppliers to consumers, minimizing the total cost of transportation:

$$
L_{1}(X)=\sum_{i=1}^{m} \sum_{j=1}^{n} c_{i j} x_{i j}
$$

and satisfying the constraints:

$$
\begin{gathered}
\sum_{j=1}^{n} x_{i j} \leq a_{i}, \quad i=1,2, \ldots, m, \\
\sum_{i=1}^{m} x_{i j} \geq b_{j}, \quad j=1,2, \ldots, n, \\
x_{i j} \geq 0, \quad i=1,2, \ldots, m, \quad j=1,2, \ldots, n .
\end{gathered}
$$

By adding dummy variables, this problem is reduced to the canonical one, in which inequalities (2), (3) are transformed into equalities. Methods for solving this problem are well known and implemented in widely used mathematical packages (Mathcad, Excel, etc.)

In a market economy, the above formulation of the transport problem cannot be recognized as realistic, since the parameters of the problem are not deterministic values. In practice, the uncertainty of the initial data can manifest itself in the random nature of the capabilities of suppliers, the needs of consumers (demand for a product), and the cost of transportation. It is clear that under these conditions the solution of the problem must also contain uncertainty and its obtaining by traditional methods is impossible.

In well-known works, specific tasks of the arisen general problem are solved. In [4] the method of accounting for the uncertainty in the state of vehicles is considered, in [5] the problem of forming routes by the methods of fuzzy logic was solved. A similar approach was used to solve three-index distribution control problems in transport networks [6,7]. In [8], an overview of the formulations of transport-type problems arising in connection with the uncertainty of the initial data is given.

Methods for solving such problems are combined into a specific subclass of general methods of mathematical programming, called stochastic programming [9-13]. The technology for solving stochastic programming problems consists in constructing for each of them a corresponding deterministic analogue using further standard methods of mathematical programming. Moreover, as an objective function in such problems, the mathematical expectation or variance of the random value of the total cost of transportation is usually used [11-13].

A productive attempt to solve this problem using a more informative objective function was implemented in [14] in the most studied version of this problem. In this case, it was assumed that the cost of transportation is random values. Further, it is assumed that there is enough real data to formulate plausible hypotheses about the distribution laws of these quantities and accept them statistically soundly. A large number of various factors independently affect the cost of transporting goods. In this regard, in practice, it is usually believed that their joint influence, in accordance with the central limit theorem of probability theory, leads to the normality of the resulting random variables.

In accordance with this, a set of densities:

$$
\phi_{i j}\left(c_{i j}\right)=\frac{1}{\sqrt{2 \pi} \sigma_{j}} \exp \left\{-\frac{\left(c_{i j}-m_{i j}\right)^{2}}{2 \sigma_{i j}^{2}}\right\}, \quad i=1,2, \ldots m, \quad j=1,2, \ldots, n .
$$

Then the distribution density of a random variable of the total cost of transportation:

$$
R=L(X)=\sum_{i=1}^{m} \sum_{j=1}^{n} c_{i j} x_{i j}
$$


has the form:

$$
f(R)=f(L(X))=\frac{1}{\sqrt{2 \pi} \sigma_{\Sigma}} \exp \left\{-\frac{\left(R-m_{\Sigma}\right)^{2}}{2 \sigma_{\Sigma}^{2}}\right\}
$$

where

$$
m_{\Sigma}=\sum_{i={ }^{\prime}}^{m} \sum_{j=1}^{n} m_{i j} x_{i j}, \quad \sigma_{\Sigma}^{2}=\sum_{i={ }^{\prime}}^{m} \sum_{j=1}^{n} \sigma_{i j}^{2} x_{i j}^{2} .
$$

The problem is to find a set $X=\left(x_{i j}\right)$ that delivers an extremal value of some naturally chosen function of density $f(R)$ and satisfies constraints (2)-(4). In this case, for example, let's set a certain threshold value $R_{t}$ of the total cost of transportation, the excess of which is regarded as a sign of inefficiency of the corresponding transportation plan. Then, as the objective function, it is natural to choose the probability that the total cost of transportation will exceed the threshold. This probability is:

$$
P\left(R \geq R_{n}\right)=\int_{R_{T}}^{\infty} f(R) \mathrm{d} R=\int_{R_{T}}^{\infty} \frac{1}{\sqrt{2 \pi} \sigma_{\Sigma}} \exp \left\{-\frac{\left(R-m_{\Sigma}\right)^{2}}{2 \sigma_{\Sigma}^{2}}\right\} \mathrm{d} R \underset{\frac{R-m_{\Sigma}}{\sigma_{\Sigma}}=u}{=} \frac{1}{\sqrt{2 \pi}} \int_{\frac{R_{T}-m_{\Sigma}}{\sigma_{\Sigma}}}^{\infty} e^{-\frac{u^{2}}{2}} \mathrm{~d} u .
$$

Now the original problem is transformed to the following: find a transportation plan $X$ satisfying the constraints and minimizing (5).

It is clear that minimization (5) is equivalent to maximizing the lower limit in this integral, equal to:

$$
R(X)=\frac{R_{T}-m_{\Sigma}}{\sigma_{\Sigma}}=\frac{R_{T}-\sum_{i=1}^{m} \sum_{j=1}^{n} m_{i j} x_{i j}}{\left(\sum_{i=1}^{m} \sum_{j=1}^{n} \sigma_{i j}^{2} x_{i j}^{2}\right)^{\frac{1}{2}}} .
$$

Let's introduce the parameter $A=\sum_{i=1}^{m} a_{i}$.

If the transportation plan $X$ satisfies constraints (2) as equalities, then:

$$
A=\sum_{i=1}^{m} a_{i}=\sum_{i=1}^{m} \sum_{j=1}^{n} x_{i j}
$$

Then (6) can be transformed to the form:

$$
R(X)=\frac{\frac{R_{n}}{A} \sum_{i=1}^{m} \sum_{j=1}^{n} x_{i j}-\sum_{i=1}^{m} \sum_{j=1}^{n} m_{i j} x_{i j}}{\left(\sum_{i=1}^{m} \sum_{j=1}^{n} \sigma_{i j}^{2} x_{i j}^{2}\right)^{\frac{1}{2}}}=\frac{\sum_{i=1}^{m} \sum_{j=1}^{n}\left(\frac{R_{n}}{A}-m_{i j}\right) x_{i j}}{\left(\sum_{i=1}^{m} \sum_{j=1}^{n} \sigma_{i j}^{2} x_{i j}^{2}\right)^{\frac{1}{2}}}=\frac{\sum_{i=1}^{m} \sum_{j=1}^{n} d_{i j} x_{i j}}{\left(\sum_{i=1}^{m} \sum_{j=1}^{n} \sigma_{i j}^{2} x_{i j}^{2}\right)^{\frac{1}{2}}},
$$

where $d_{i j}=\frac{R_{n}}{A}-m_{i j}, i=1,2, \ldots m, j=1,2, \ldots, n$.

The obtained maximization problem (7) with satisfaction (2)-(4) is a complex problem of fractional-nonlinear programming. In [14], an approximate solution to this problem is proposed, obtained as follows.

Because:

$$
\left(\sum_{i=1}^{m} \sum_{j=1}^{n} \sigma_{i j}^{2} x_{i j}^{2}\right)^{\frac{1}{2}}<\left(\sum_{i_{1}=1}^{m} \sum_{j_{1}=1}^{n} \sum_{i_{2}=1}^{m} \sum_{j_{2}=1}^{n} \sigma_{i_{1} j_{1}} \sigma_{i_{2} j_{2}} x_{i_{1} j_{1}} x_{i_{2} j_{2}}\right)^{\frac{1}{2}}=\left[\left(\sum_{i=1}^{m} \sum_{j=1}^{n} \sigma_{i j} x_{i j}\right)^{2}\right]^{\frac{1}{2}}=\sum_{i=1}^{m} \sum_{j=1}^{n} \sigma_{i j} x_{i j},
$$


then

$$
R(X)=\frac{\sum_{i=1}^{m} \sum_{j=1}^{n} d_{i j} x_{i j}}{\left(\sum_{i=1}^{m} \sum_{j=1}^{n} \sigma_{i j}^{2} x_{i j}^{2}\right)^{\frac{1}{2}}}>\frac{\sum_{i=1}^{m} \sum_{j=1}^{n} d_{i j} x_{i j}}{\sum_{i=1}^{m} \sum_{j=1}^{n} \sigma_{i j} x_{i j}}=T(X) .
$$

In this case, the problem is reduced to finding a plan $X$ satisfying the constraints and maximizing $T(X)$. By virtue of (8), on the set of plans $X$, the values of the function $T(X)$ minor the values of the function $R(X)$, that is, the process of maximizing the lower bound for $R(X)$ is accompanied by an increase in the value of $R(X)$, which is required. To solve the problem, it is proposed to use the following iterative procedure.

An arbitrary design $X^{(0)}$ is chosen that satisfies constraints (2)-(4). If this design does not maximize $T(X)$, then there must be some other design $X^{(1)}$ for which:

$$
T\left(X^{(1)}\right)-T\left(X^{(0)}\right)>0
$$

Since $\sum_{i=1}^{m} \sum_{j=1}^{n} \sigma_{i j} x_{i j}>0$ on any design of the problem, it follows from (9) that:

$$
\begin{gathered}
\left(T\left(X^{(1)}\right)-T\left(X^{(0)}\right)\right)\left(\sum_{i=1}^{m} \sum_{j=1}^{n} \sigma_{i j} x_{i j}^{(1)}\right)=\sum_{i=1}^{m} \sum_{j=1}^{n} d_{i j} x_{i j}^{(1)}-T\left(X^{(0)}\right) \sum_{i=1}^{m} \sum_{j=1}^{n} \sigma_{i j} x_{i j}^{(1)}= \\
=\sum_{i=1}^{m} \sum_{j=1}^{n}\left(d_{i j}-T\left(X^{(0)}\right) \sigma_{i j}\right) x_{i j}^{(1)}=\sum_{i=1}^{m} \sum_{j=1}^{n} d_{i j}^{(1)} x_{i j}^{(1)}>0 .
\end{gathered}
$$

Now the task is to find a design $X^{(1)}$ that maximizes:

$$
S\left(X^{(1)}\right)=\sum_{i=1}^{m} \sum_{j=1}^{n} d_{i j}^{(1)} x_{i j}^{(1)}
$$

and satisfying constraints (2)-(4). An ordinary transport linear programming problem is obtained. If the design $X^{(1)}$ calculated as a result of its solution does not maximize $T(X)$, then there must exist a new design $X^{(2)}$ for which $T\left(X^{(2)}\right)-T\left(X^{(1)}\right)>0$.

Thus, the original problem is reduced to an iterative procedure for finding a sequence of designs $X^{*(1)}, X^{*(2)}, \ldots, X^{*}(k), X^{*}(k+1)$ satisfying the constraints for which the recurrence relation:

$$
\begin{gathered}
\sum_{i=1}^{m} \sum_{j=1}^{n} d_{i j} x_{i j}^{*(k+1)}-T\left(X^{*(k)}\right) \sum_{i=1}^{m} \sum_{j=1}^{n} \sigma_{i j} x_{i j}^{*(k+1)}=\max _{X^{(k+1)}}\left[\sum_{i=1}^{m} \sum_{j=1}^{n} d_{i j} x_{i j}^{*(k+1)}-T\left(X^{*(k)}\right) \sum_{i=1}^{m} \sum_{j=1}^{n} \sigma_{i j} x_{i j}^{*(k+1)}\right]= \\
=\max _{X^{(k+1)}} \sum_{i=1}^{m} \sum_{j=1}^{n}\left(d_{i j}-T\left(X^{*(k)}\right) \sigma_{i j}\right) x_{i j}^{(k+1)}=\max _{X^{\prime(k+1)}} \sum_{i=1}^{m} \sum_{j=1}^{n} d_{i j}^{(k+1)} x_{i j}^{(k+1)} .
\end{gathered}
$$

It is natural to stop the computational procedure when the inequality:

$$
\sum_{i=1}^{m} \sum_{j=1}^{n}\left|x_{i j}^{(k+1)}-x_{i j}^{(k)}\right|<\varepsilon
$$

is satisfied, where $\varepsilon-\mathrm{a}$ small number.

It is clear that with increasing $T(X)$, in accordance with (8), $R(X)$ will grow. On the other hand, a design that maximizes $T(X)$ does not necessarily maximize $R(X)$. In this case, the degree of approximation to the exact solution of the problem is not clear. 
In [14], it was noted that in the particular case, when the variances of all delivery costs for a unit of goods are equal, the problem of finding the required design is simplified to the usual transport one. Indeed, let $\sigma_{i j}^{2}=\sigma^{2}, i=1,2, \ldots, m, j=1,2, \ldots, n$. Then the expression for $T(X)$ is simplified to the form:

$$
T(X)=\frac{\sum_{i=1}^{m} \sum_{j=1}^{n} d_{i j} x_{i j}}{\sum_{i=1}^{m} \sum_{j=1}^{n} \sigma_{i j} x_{i j}}=\frac{\sum_{i=1}^{m} \sum_{j=1}^{n}\left(\frac{R_{n}}{A}-m_{i j}\right) x_{i j}}{\sigma \sum_{i=1}^{m} \sum_{j=1}^{n} x_{i j}}=\frac{R_{n}-\sum_{i=1}^{m} \sum_{j=1}^{n} m_{i j} x_{i j}}{\sigma \cdot A}=\frac{R_{n}}{\sigma \cdot A}-\frac{1}{\sigma \cdot A} \sum_{i=1}^{m} \sum_{j=1}^{n} m_{i j} x_{i j} .
$$

It is clear that maximizing the resulting expression is equivalent to minimizing:

$$
C(X)=\sum_{i=1}^{m} \sum_{j=1}^{n} m_{i j} x_{i j}
$$

At the same time, an ordinary transport linear programming problem was obtained, the solution of which gives an approximate solution to the original problem (2)-(4), (7).

A brief analysis of publications on the problem of solving transport problems in conditions of uncertainty allows to draw the following conclusion.

In the formulated problem of calculating the transportation plan under conditions of their random cost, there is no method for obtaining an exact solution. The quality of the approximate solution obtained using (8), as well as the rate of its convergence, cannot be estimated. In addition, it is completely unclear how to solve the formulated problems when the distribution density of the random cost of transportation is not known.

The aim of research is to develop mathematical models and effective methods for solving transport problems in conditions of uncertainty. To achieve the aim, it is necessary to solve the following objectives:

1. Development of effective (accurate and fast) methods for solving transport problems with a probabilistic description of the cost of transportation.

2. Development of a method for solving transport problems for the case when the distribution density of the random cost of transportation is not known.

\section{Problem statement}

Let the mathematical model of the transport problem under uncertainty have a canonical representation (1)-(5) and the corresponding optimization problem be formulated as follows: find a plan $X$ that maximizes the fractional - quadratic function (11) and satisfies constraints (2)-(4).

\section{Research results}

\section{1. A method for solving transport problems in the context of a probabilistic descrip-} tion of the cost of transportation

Any exact solution to problem (8), (2)-(4) can be obtained by solving a recurrent sequence of nonlinear programming problems. It is clear that the maximum $R(X)$ is reached on the same set as the maximum $R^{2}(X)$. In accordance with this, let's obtain:

$$
F(X)=R^{2}(X)=\frac{\left(\sum_{i=1}^{m} \sum_{j=1}^{n} d_{i j} x_{i j}\right)^{2}}{\sum_{i=1}^{m} \sum_{j=1}^{n} \sigma_{i j}^{2} x_{i j}^{2}} .
$$

Thus, the problem is reduced to the following: find a design $X$ that maximizes the fractional-quadratic function (11) and satisfies constraints (2)-(4). It is proposed to solve this problem as follows. 
Introduce columns $C, X$ and matrix $G$ :

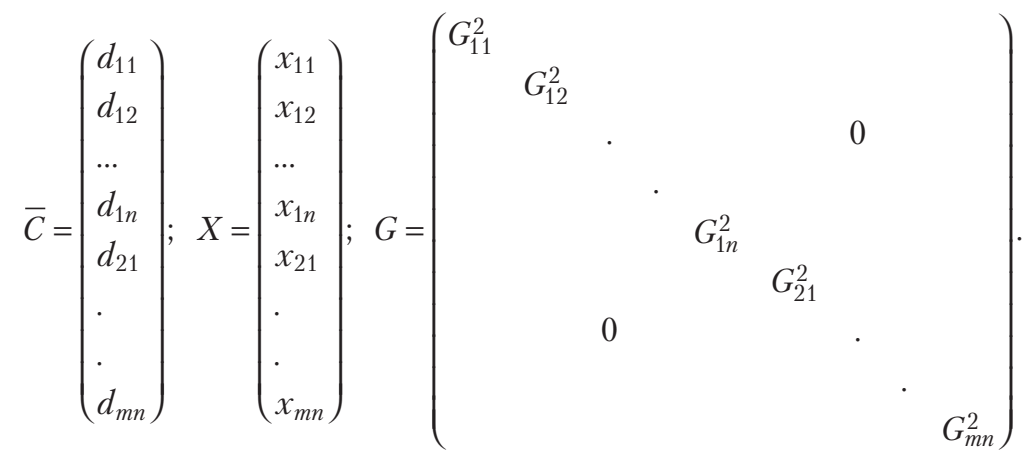

Then relation (11) takes the form:

$$
\begin{gathered}
L(X)=\frac{X^{T} \bar{C} \cdot \bar{C}^{T} X}{X^{T} G X}=\frac{X^{T} C X}{X^{T} G X}, \\
C=\bar{C} \bar{C}^{T} .
\end{gathered}
$$

Now the problem is reduced to finding a set $X$ that maximizes (12) and satisfies:

$$
A X=B,
$$

where (13) is a matrix analogue of (2)-(4).

$$
X \geq 0 .
$$

The solution to this problem is achieved by implementing the following iterative procedure, similar to that described above. An arbitrary vector $X^{(0)}$ is chosen, which is a solution to the system of equations $A X=B$ and satisfies the constraint $X \geq 0$. If this vector is not a solution to the problem, that is, does not maximize (12), then there must be some other vector $X^{(1)}$ for which:

$$
L\left(X^{(1)}\right)-L\left(X^{(0)}\right)>0 .
$$

Since $X^{T} D X>0$, it follows from (15) that:

$$
\left(L\left(X^{(1)}\right)-L\left(X^{(0)}\right)\right) X^{(1) T} G X^{(1)}=X^{(1) T} C X^{(1)}-X^{(1) T} G X^{(1)} L\left(X^{(0)}\right) \geq 0 .
$$

Taking into account (16), the problem is reduced to finding a vector $X^{(1)}$ satisfying (13), (14) and maximizing:

$$
R\left(X^{(1)}\right)=X^{(1) T}\left(C-L\left(X^{(0)}\right) G\right) X^{(1)} .
$$

If the resulting vector does not maximize (12), then there must exist some other vector $X^{(2)}$ for which:

$$
L\left(X^{(2)}\right)-L\left(X^{(1)}\right)>0 .
$$

Thus, the original problem is reduced to an iterative procedure for finding a sequence of vectors satisfying (13), (14):

$$
X^{*(1)}, X^{*(2)}, \ldots, X^{*(k)}, X^{*(k+1)},
$$


for which the recurrence relation holds:

$$
\begin{gathered}
X^{*(k+1) T} C X^{*(k+1)}-X^{*(k+1) T} G X^{*(k+1)} L\left(X^{*(k)}\right)= \\
=\max _{X^{(k+1)}}\left[X^{(k+1) T} C X^{(k+1)}-X^{(k+1) T} G X^{(k+1)} L\left(X^{*(k)}\right)\right]= \\
=\max _{X^{(k+1)}} X^{(k+1) T}\left(C-G L\left(X^{*(k)}\right)\right) X^{(k+1)}=\max _{X^{(k+1)}} X^{(k+1) T} G_{k} X^{k+1} .
\end{gathered}
$$

It is natural to stop the computational procedure when the inequality $\left\|X^{(k+1)}-X^{(k)}\right\|<\varepsilon$ is satisfied, where $\varepsilon$-some sufficiently small number. This ensures the required accuracy of solving the problem.

Each of the sequence of problems (17) is much easier than the original problem, since here the maximization of the fractional-quadratic functional (12) is replaced by the optimization of the usual quadratic functional (17). Moreover, at each iteration, it is necessary to solve problems of the form: find a vector $X$ that maximizes:

$$
f(x)=X^{T} G X
$$

and satisfying:

$$
\begin{gathered}
A X=B, \\
X \geq 0 .
\end{gathered}
$$

The Lagrange function is formed:

$$
F(X, \Lambda)=f(x)-\Lambda^{T}(A X-B)=X^{T} G X-\Lambda^{T}(A X-B) .
$$

As is known, if $X^{*}$ - the optimal solution to problem (18)-(21), then there must exist a vector $\Lambda^{*}$ such that $X^{*}$ and $\Lambda^{*}$ satisfy the relations:

$$
\begin{gathered}
\left.\frac{d F(X, \Lambda)}{d X}\right|_{\substack{X=X^{*} \\
\Lambda=\Lambda^{*}}}=2\left(X^{*}\right)^{T} G-\Lambda^{* T} A \leq 0, \\
\frac{d F\left(X^{*}, \Lambda^{*}\right)}{d X} X^{*}=0, \\
\frac{d F\left(X^{*}, \Lambda^{*}\right)}{d \Lambda} X^{*}=B-A X=0 .
\end{gathered}
$$

Transposing (24):

$$
2 G X^{*}-A^{T} \Lambda \leq 0
$$

or

$$
A^{T} \Lambda-2 G X^{*} \geq 0 \text {. }
$$

Let's introduce the vector:

$$
V^{*}=A^{T} \Lambda-2 G X^{*} \geq 0
$$


Then, taking into account (25),

$$
2 G X^{*}-A^{T} \Lambda+V^{*}=0 .
$$

Further, let's write (23) as follows:

$$
\left(V^{*}\right)^{T} X^{*}=0
$$

or

$$
\left(X^{*}\right)^{T} V^{*}=0 .
$$

Thus, if $X^{*}$ is a solution to the problem, then there exist $V^{*} \geq 0$ and $\Lambda^{*}$ such that relations (24), (26), (27) hold. On the other hand, if there exist $X \geq 0, V^{*} \geq 0, \Lambda$, which satisfy the conditions:

$$
A X=B, 2 G X-A^{T} \Lambda+V=0, \quad X^{T} V=0
$$

then the vector $X$ is the optimal solution to the problem with (18)-(20).

Let's write the first two equations of system (28) in matrix form:

$$
\left(\begin{array}{ccc}
A & 0 & 0 \\
2 G & -A^{T} & I
\end{array}\right)\left(\begin{array}{l}
X \\
\Lambda \\
V
\end{array}\right)=\left(\begin{array}{l}
B \\
0
\end{array}\right) .
$$

Let's note that a solution to system (29) that satisfies the requirements $X \geq 0, V \geq 0, X^{T} V=0$, has at most $m+n$ nonzero components. Indeed, if $k$ components of vector $X$ are positive, then, in view of $X^{T} V=0$, at least the same number of components of vector $V$ are equal to zero. Therefore, at most $n-k$ components of the vector $V$ are positive. Therefore, in a $2 n$-dimensional vector, at most components are positive. Since there is a dimensional vector, the vector contains at most $n+m$ nonzero components. It follows that a vector satisfying conditions (28) for $X \geq 0, V \geq 0$ must be a basic solution to system (29), that is, it contains at most $n+m$ positive components.

The procedure for obtaining a vector $\left(X^{T} V^{T} \Lambda^{T}\right)$ satisfying (23) is as follows. First, let's find the basic solution of the system $A X=B$ using the usual first step of the simplex method of linear programming. Let $A_{0}$ be a square $\mathrm{m} \times \mathrm{m}$ submatrix of the matrix $A$ whose columns correspond to the basic (nonzero) variables that form the vector $X_{0}$. In this case, naturally, $A_{0} X_{0}=b$.

Note that the basic solution $X_{0}$ satisfies the first m constraints of system (29). Therefore, the further solution is associated with finding an admissible n-component vector $V$ that, together with $X$, satisfies the rest of the equations of system (29). This vector can be obtained by solving the following modified linear programming problem: find a vector satisfying the system of equations:

$$
A X=B, \quad 2 G X-A^{T} \Lambda+V+E U=0
$$

and minimizing the linear shape:

$$
L(U)=E U, E=\left(\begin{array}{lll}
1 & & 0 \\
& \ldots & \\
0 & & 1
\end{array}\right),
$$

depending on the components of the artificial basis $U^{T}=\left(u_{1}, u_{2}, \ldots, u_{n}\right)$. The solution of the problem ends after the derivation of the artificial variables $U$ from the basis. The difference between the problem being solved and the usual linear programming problem is that no pair of components $\left(x_{j} v_{j}\right)$ that are introduced into the basis can simultaneously contain both positive components.

A significant drawback of this technique is the slow convergence of the procedure for finding a sequence of solutions $X^{*(1)}, X^{*(2)}, \ldots, X^{*(k)}$ in problem (15)-(17). This noted feature is explained by the fact that at each iteration of the procedure for sequential improvement of the objective function (12), it is necessary to solve a quadratic programming problem, which is difficult in itself. In this case, only 
an approximate estimate of the number of iterations required to obtain an admissible solution in terms of accuracy is possible. In this regard, let's consider another method for solving the problem.

It is convenient to replace the problem of maximizing function (11) by the equivalent problem of minimizing the function:

$$
G(x)=\frac{\sum_{i=1}^{m} \sum_{j=1}^{n} \sigma_{i j}^{2} x_{i j}^{2}}{\left(\sum_{i=1}^{m} \sum_{j=1}^{n} d_{i j} x_{i j}\right)^{2}} .
$$

To solve the fractional-quadratic programming problem obtained in this form, let's introduce a new variable:

$$
y_{0}=\frac{1}{\sum_{i=1}^{m} \sum_{j=1}^{n} d_{i j} x_{i j}}
$$

From where:

$$
y_{0} \sum_{i=1}^{m} \sum_{j=1}^{n} d_{i j} x_{i j}=1
$$

Let's formulate a new set of variables:

$$
y_{i j}=y_{0} x_{i j}, \quad i=1,2, \ldots, m, \quad j=1,2, \ldots, n \text {. }
$$

In this case, relations (2)-(4), (31), (32) take the form:

$$
\begin{gathered}
G(y)=\sum_{i=1}^{m} \sum_{j=1}^{n} \sigma_{i j}^{2} y_{i j}^{2}, \\
\sum_{i=1}^{m} \sum_{j=1}^{n} d_{i j} y_{i j}=1, \\
\sum_{j=1}^{n} x_{i j}=\frac{1}{y_{0}} \sum_{j=1}^{n} y_{i j}=a_{i}, \quad i=1,2, \ldots, m, \\
\sum_{i=1}^{m} x_{i j}=\frac{1}{y_{0}} \sum_{i=1}^{m} y_{i j}=b_{j}, \quad j=1,2, \ldots, n,
\end{gathered}
$$

From where:

$$
\begin{aligned}
& \sum_{j=1}^{n} y_{i j}=y_{0} a_{i}, \quad i=1,2, \ldots, m, \\
& \sum_{i=1}^{m} y_{i j}=y_{0} b_{j}, \quad j=1,2, \ldots, n .
\end{aligned}
$$

Now the original problem has been reduced to the form: find a set $Y=\left(y_{i j}\right), i=1,2, \ldots, m$, $j=1,2, \ldots, n$, minimizing (34) and satisfying constraints (35)-(37). Let's solve the resulting quadratic programming problem.

Let's introduce the Lagrange function:

$$
\Phi(y)=\sum_{i=1}^{m} \sum_{j=1}^{n} \sigma_{i j}^{2} y_{i j}^{2}-\sum_{i=1}^{m} \lambda_{i}\left(\sum_{j=1}^{n} y_{i j}-y_{0} a_{i}\right)-\sum_{j=1}^{n} \mu_{j}\left(\sum_{i=1}^{m} y_{i j}-y_{0} b_{j}\right) .
$$


Further:

$$
\begin{gathered}
\frac{d \Phi(y)}{d y_{i j}}=2 \sigma_{i j}^{2} y_{i j}-\lambda_{i}-\mu_{j}=0, \quad i=1,2, \ldots, m, \quad j=1,2, \ldots, n, \\
y_{i j}=\frac{1}{2 \sigma_{i j}^{2}}\left(\lambda_{i}+\mu_{j}\right), \quad i=1,2, \ldots, m, \quad j=1,2, \ldots, n .
\end{gathered}
$$

Substituting (38) into (35)-(37),

$$
\begin{aligned}
& \sum_{j=1}^{n} \frac{1}{2 \sigma_{i j}^{2}}\left(\lambda_{i}+\mu_{j}+v d_{i j}\right)=y_{0} a_{i}, \quad i=1,2, \ldots, m, \\
& \sum_{i=1}^{m} \frac{1}{2 \sigma_{i j}^{2}}\left(\lambda_{i}+\mu_{j}+v d_{i j}\right)=y_{0} b_{j}, \quad j=1,2, \ldots, n .
\end{aligned}
$$

Solving the resulting system of linear algebraic equations, let's obtain expressions for $\left\{\lambda_{i}\right\}$, $\left\{\mu_{j}\right\}$ in terms of $y_{0}$ and the initial data $\left\{a_{i}\right\},\left\{b_{j}\right\}$. Substituting these expressions in (38), let's obtain the relations for $y_{i j}$ in terms of $y_{0}$. Now let's find the value $y_{0}$ from (34). Finally, let's calculate the values of the initial variables $x_{i j}$ using (33). The problem has been solved.

As an example of the application of the proposed method, consider the following, maximally simple transport problem. Let there are two points of delivery of a certain product in quantities $a_{1}$ and $a_{2}$ units, as well as two points of consumption of this product in the amount of $b_{1}$ and $b_{2}$ units. Let's set the parameters of the corresponding transport network: $a_{1}=120, a_{2}=80, b_{1}=60, b_{2}=140$ and the matrix of average costs of transportation of a unit of cargo from suppliers to consumers:

$$
M=\left(\begin{array}{ll}
m_{11} & m_{12} \\
m_{21} & m_{22}
\end{array}\right)=\left(\begin{array}{ll}
11.5 & 10 \\
10.5 & 11
\end{array}\right) .
$$

Let's introduce the required matrix $X=\left(x_{i j}\right)$ defining the transportation plan. In this case, the objective function of the problem - the average total cost of transportation has the form:

$$
\bar{R}(x)=\sum_{i=1}^{2} \sum_{j=1}^{2} m_{i j} x_{i j},
$$

and the restrictions on the sought variables $x_{i j}$ are determined by the expressions:

$$
\begin{aligned}
& x_{11}+x_{12}=a_{1}=120, \\
& x_{21}+x_{22}=a_{2}=80, \\
& x_{11}+x_{21}=b_{1}=60, \\
& x_{12}+x_{22}=b_{2}=140 .
\end{aligned}
$$

It is required to find a design $X=\left(x_{i j}\right)$ that minimizes the objective function $\bar{R}(x)$ satisfying the constraints. The solution to this simplest problem, found by the method of the minimum element of the matrix $M$, has the form:

$$
\begin{aligned}
& x_{11}{ }^{(0)}=0, \\
& x_{12}{ }^{(0)}=120, \\
& x_{21}{ }^{(0)}=60, \\
& x_{22}{ }^{(0)}=20 .
\end{aligned}
$$

The objective function value corresponding to the received transportation plan $X^{(0)}$ is:

$$
\bar{R}\left(X^{(0)}\right)=120 \cdot 10+60 \cdot 10.5+20 \cdot 11=2050 \text { units. }
$$


Let's now consider a more informative version of the formulation of this problem, in which the matrix of variances of random costs of transportation of a unit of cargo is given:

$$
\sigma^{2}=\left(\begin{array}{ll}
\sigma_{11}^{2} & \sigma_{12}^{2} \\
\sigma_{21}^{2} & \sigma_{22}^{2}
\end{array}\right)=\left(\begin{array}{cc}
8 & 10 \\
12 & 6
\end{array}\right)
$$

Let's solve the problem of finding a transportation plan that satisfies the constraints that minimizes the probability that the random value of the total cost of transportation will exceed a given threshold value. In accordance with the proposed technique, the Lagrange function is formed:

$$
\phi(y)=\sum_{i=1}^{2} \sum_{j=1}^{2} \sigma^{2} i j y^{2} i j-\sum_{i=1}^{2} \lambda_{i}\left(\sum_{j=1}^{2} y_{i j}-y_{0} a_{i}\right)-\sum_{j=1}^{2} \mu_{j}\left(\sum_{i=1}^{2} y_{i j}-b_{j}\right)
$$

and the system of linear algebraic equations is solved for the introduced variables $y_{i j}$ These equations have the form:

$$
2 \sigma^{2}{ }_{i j} y_{i j}-\lambda_{i}-\mu_{j}=0, i=1,2 ; j=1,2
$$

From where:

$$
y_{i j}=\frac{1}{2 \sigma^{2} i j}\left(\lambda_{i}+\mu_{j}\right), i=1,2 ; j=1,2 .
$$

Substituting the obtained expressions for $y_{i j}$ and the numerical values of the problem parameters into the equations for the constraints, let's obtain the following system of algebraic equations:

$$
\begin{aligned}
& y_{11}+y_{12}=\frac{1}{16}\left(\lambda_{1}+\mu_{1}\right)+\frac{1}{20}\left(\lambda_{1}+\mu_{2}\right)=120 y_{0}, \\
& y_{21}+y_{22}=\frac{1}{24}\left(\lambda_{2}+\mu_{1}\right)+\frac{1}{12}\left(\lambda_{2}+\mu_{2}\right)=80 y_{0}, \\
& y_{11}+y_{21}=\frac{1}{16}\left(\lambda_{1}+\mu_{1}\right)+\frac{1}{24}\left(\lambda_{2}+\mu_{1}\right)=60 y_{0}, \\
& y_{12}+y_{22}=\frac{1}{20}\left(\lambda_{1}+\mu_{2}\right)+\frac{1}{12}\left(\lambda_{2}+\mu_{2}\right)=140 y_{0} .
\end{aligned}
$$

After reducing similar terms, the system takes the following form:

$$
\begin{array}{ccccc}
0.1125 \lambda_{1} & & +0.0625 \mu_{1} & +0.050 \mu_{2} & =120 y_{0}, \\
& 0.125 \lambda_{2} & +0.042 \mu_{1} & +0.0833 \mu_{2} & =80 y_{0}, \\
0.0625 \lambda_{1} & +0.0417 \lambda_{2} & +0.104 \mu_{1} & & =60 y_{0}, \\
0.05 \lambda_{1} & +0.084 \lambda_{2} & & +0.1333 \mu_{2} & =140 y_{0} .
\end{array}
$$

The solution to this system gives a description of the parameters $\lambda_{i}, \mu_{j}$ in terms of $y_{0}$ :

$$
\lambda_{1}=600 y_{0}, \lambda_{2}=40 y_{0}, \mu_{1}=200 y_{0}, \mu_{2}=800 y_{0} .
$$

From where:

$$
\begin{aligned}
& y_{11}=\frac{1}{16}\left(\lambda_{1}+\mu_{1}\right)=50 y_{0}, \\
& y_{12}=\frac{1}{20}\left(\lambda_{1}+\mu_{2}\right)=70 y_{0}, \\
& y_{21}=\frac{1}{24}\left(\lambda_{2}+\mu_{1}\right)=10 y_{0}, \\
& y_{22}=\frac{1}{12}\left(\lambda_{2}+\mu_{2}\right)=70 y_{0} .
\end{aligned}
$$


Now, taking into account (33), let's find the required transportation design:

$$
\begin{aligned}
& x_{11}{ }^{(1)}=50, \\
& x_{12}{ }^{(1)}=70, \\
& x_{21}{ }^{(1)}=10, \\
& x_{22}{ }^{(1)}=70 .
\end{aligned}
$$

The solution is over.

Now it is useful to evaluate the merit of the proposed methodology for optimizing the transportation plan under conditions of uncertainty. For this purpose, let's compare the probabilities of exceeding the threshold value by a random value of the total cost of transportation. As a threshold, let's choose a value that exceeds the average total cost of transportation by $15 \%$. For the plan $X^{(0)}$, obtained by optimization on average without taking into account the variances of the cost of transportation, let's obtain:

$$
\begin{gathered}
R_{0}=\sum_{i=1}^{2} \sum_{j=1}^{2} m_{i j} x_{i j}^{(0)} 10 * 120+11 * 20+10.5 * 60=2050, \\
\sigma_{\Sigma}^{(0)}=\left(\sum_{i=1}^{2} \sum_{j=1}^{2} \sigma_{i j}^{2}\left(x_{i j}^{(0)}\right)^{2}\right)^{\frac{1}{2}}=\left[10 \times(120)^{2}+6 \times(20)^{2}+12 \times(60)^{2}\right]^{\frac{1}{2}}=435.4, \\
\operatorname{Bep}\left\{R_{0} \geq R_{n}\right\}=\operatorname{Bep}\left\{R_{0} \geq 1.15 \overline{R_{0}}\right\}=0.175 .
\end{gathered}
$$

For the plan $X^{(1)}$, obtained taking into account the variances of the cost of transportation, let's obtain:

$$
\begin{gathered}
\overline{R_{1}}=\sum_{i=1}^{2} \sum_{j=1}^{2} m_{i j} x_{i j}^{(1)}=11.5 \times 50+10 \times 70+10.5 \times 70+11 \times 70=2150, \\
\sigma_{\Sigma}^{(1)}=\left(\sum_{i=1}^{2} \sum_{j=1}^{2} \sigma_{i j}^{2}\left(x_{i j}^{(1)}\right)^{2}\right)^{\frac{1}{2}}=8 \times(50)^{2}+10 \times(70)^{2}+6 \times(70)^{2}+12 \times(10)^{2}=315.2, \\
\operatorname{Bep}\left\{R_{1} \geq R_{n}\right\}=\operatorname{Bep}\left\{R_{1} \geq 1.15 \overline{R_{1}}\right\}=0.075 .
\end{gathered}
$$

Thus, the probability of exceeding the permissible threshold in this case is two times less than the previous one. The apparent advantage of the proposed method, naturally, will be the greater, the higher the differences in the variances of the cost of transportation for different routes and the greater the dimension of the problem.

Let's note that the fundamental advantage of the proposed method for solving a transport problem under uncertainty is the ability to obtain an accurate and fast result due to a single solution of the fractional-square programming problem.

The real complication of the problem arises if the statistical data for adequate reconstruction of the distribution density of the random costs of transportation is not enough, but a correct statistical estimate of the mathematical expectations and variances of these random variables is possible.

3. 2. A method for solving transport problems for the case when the distribution density of the random cost of transportation is not known

Let $m_{i j}$ and $\sigma_{i j}^{2}$ be statistical estimates of the mathematical expectation and variance of the random cost of transporting a unit of product from the $i$-th supplier to the $j$-th consumer. To solve the problem, the following two-stage procedure is proposed. 
At the first stage, let's find a transportation plan X that minimizes the average total cost of transportation:

$$
M[L(x)]=\sum_{i=1}^{m} \sum_{j=1}^{n} m_{i j} x_{i j}
$$

and satisfying constraints (2)-(4). This is a common transportation problem and let $X^{(0)}$ be its solutions.

At the second stage, let's pose the problem of finding a plan that minimizes the trade-off criterion composed of four terms. The first term determines the variance of the total cost of transportation, the second - the measure of deviation of the desired plan from the plan $X^{(0)}$, the third term provides a solution that satisfies (2), the fourth - satisfaction (3). Thus, the composite criterion has the form

$$
L(x)=\sum_{i=1}^{m} \sum_{j=1}^{n} \sigma_{i j}^{2} x_{i j}^{2}+\sum_{i=1}^{m} \sum_{j=1}^{n}\left(x_{i j}-x_{i j}^{(0)}\right)^{2}+\sum_{i=1}^{m} \lambda_{i}\left(\sum_{j=1}^{m} x_{i j}-a_{i}\right)+\sum_{j=1}^{n-1} \mu_{j}\left(\sum_{i=1}^{m} x_{i j}-b_{j}\right) .
$$

Further:

$$
\frac{d L(x)}{d x_{i j}}=2 \sigma_{i j}^{2} x_{i j}+2\left(x_{i j}-x_{i j}^{(0)}\right)+\lambda_{i}+\mu_{j}=0, \quad i=1,2, \ldots, m, \quad j=1,2, \ldots, n-1 .
$$

From where:

$$
x_{i j}=\frac{2 x_{i j}^{(0)}-\lambda_{i}-\mu_{j}}{2\left(\sigma_{i j}^{2}+1\right)}, i=1,2, \ldots, m, \quad j=1,2, \ldots, n-1
$$

Substituting (41) into (2), (3), let's obtain the system of equations:

$$
\begin{gathered}
\sum_{j=1}^{n-1} \frac{2 x_{i j}^{(0)}-\lambda_{i}-\mu_{j}}{2\left(\sigma_{i j}^{2}+1\right)}=a_{i}, \quad i=1,2, \ldots, m, \\
\sum_{i=1}^{m} \frac{2 x_{i j}^{(0)}-\lambda_{i}+\mu_{j}}{2\left(\sigma_{i j}^{2}+1\right)}=b_{j}, \quad j=1,2, \ldots, n-1 .
\end{gathered}
$$

The solution to this system of linear algebraic equations defines the sets, $\left\{\lambda_{i}\right\}, i=1,2, \ldots, m$, $\left\{\mu_{j}\right\}, j=1,2, \ldots, n-1$. Substitution of these sets in (41) gives the desired solution to the problem.

Let's now make an important remark. The results of solving the original problem naturally depend on the nature of the distribution density of the random variables $c_{i j}, i=1,2, \ldots, m$, $j=1,2, \ldots, n$. The set $\left(x_{i j}\right)$ obtained in problem (39), (40), (2), (3) corresponds to the hypothesis that this distribution is Gaussian with parameters $\left(m_{i j}, \sigma_{i j}^{2}\right)$. The real distribution can be very different from the Gaussian one. Therefore, it is advisable to consider the solution of this problem under the assumption of the worst distribution density of the random cost of transportation, having previously found this worst distribution. The problem of finding the worst distribution is solved provided that, based on the results of statistical processing of the available data for each pair $(i, j)$ «supplier - consumer», estimates of the mathematical expectation and variance of the corresponding random value $c_{i j}$ are obtained. Let's assume that the worst density corresponds to the maximum probability that the random value of the $\operatorname{cost} c_{i j}$ will exceed the given admissible threshold $c_{n}$. Thus, this problem can be formulated as follows: find the distribution density of the random cost $f\left(c_{i j}\right)$ that maximizes:

$$
p\left(c_{i j}>c_{n}\right)=\int_{c_{n}}^{\infty} f\left(c_{i j}\right) \mathrm{d} c_{i j}
$$


and satisfies the accepted restrictions:

$$
\begin{gathered}
\int_{-\infty}^{\infty} f\left(c_{i j}\right) \mathrm{d} c_{i j}=1, \\
\int_{-\infty}^{\infty} c_{i j} f(c) \mathrm{d} c=m_{i j}, \\
\int_{-\infty}^{\infty} c^{2} f(c) \mathrm{d} c=\sigma_{i j}^{2}+m_{i j}^{2} .
\end{gathered}
$$

The resulting problem is a special case of the continuous linear programming problem, the general methods of solving which are presented in [15]. It is shown that the solution to problem (42)-(45) must be sought in the form of a linear combination $\delta$ - Dirac functions. The solution procedure is iterative and consists in the sequential calculation of the components $\left(x_{1}, x_{2}, \ldots, x_{m}\right)$, $\left(\theta_{1}, \theta_{2}, \ldots, \theta_{m}\right)$ the base plan is the functions:

$$
f(\theta)=\sum_{j=1}^{m} x_{j} \delta\left(\theta-\theta_{j}\right)
$$

The plan determined at each iteration the function plan is checked for optimality. If the optimality criterion formulated in [15] is not satisfied, then the next step is taken to improve the solution. As a result of applying this procedure for problem (42)-(45), the function is obtained:

$$
f(\theta)=\frac{\left(c_{n}-m_{i j}\right)^{2}}{\sigma^{2}+\left(c_{n}-m_{i j}\right)^{2}} \delta\left(\theta-\frac{m_{i j}^{2}+\sigma^{2}-m_{i j} c_{n}}{c_{n}-m_{i j}}\right)+\frac{\sigma^{2}}{\sigma^{2}+\left(c_{n}-m_{i j}\right)^{2}} \delta\left(\theta-c_{n}\right),
$$

moreover, the probability of hitting the critical region $c>c_{n}$ is equal to the coefficient at the second term in expression (47). Thus:

$$
P\left(c>c_{n}\right)=\frac{\sigma^{2}}{\sigma^{2}+\left(c_{n}-m_{i j}\right)} .
$$

Now, using (48), an additive criterion of efficiency (expediency) of the transportation plan $X$ can be formed, taking into account the worst conditions for its implementation, which has the form:

$$
L(x)=\sum_{i=1}^{m} \sum_{j=1}^{n} \frac{\sigma_{i j}^{2}}{\sigma_{i j}^{2}+\left(c_{n}-m_{i j}\right)^{2}} x_{i j} .
$$

\section{Discussion of results}

A transport linear programming problem with random values of transportation costs is considered. The known methods for solving this problem reduce it to an optimization problem on the average. The need for a more complete account of the real uncertainty of the initial data stimulates the development of other methods for solving this problem, adequately taking into account its non-deterministic nature. To solve this problem, the following multi-step scheme is proposed. At the first step, a criterion for the optimality of the transportation plan is formulated, which sets the probability that the total random cost of transportation will exceed the permissible threshold. It is shown that the problem of finding a plan that minimizes this criterion and satisfies the system of constraints belongs to the class of fractional nonlinear programming problems. The exact method for solving such a problem is unknown. An iterative procedure for solving the problem is proposed, which transforms it at each step to a quadratic programming problem.

In order to radically accelerate the convergence of this method, an alternative version of it is proposed, which reduces the original problem to a single application of the method of indefinite Lagrange multipliers. 
The testament to the proposed scheme is the solution of the original problem for the case of a small sample of initial data. At the same time, when the required amount of statistical material to reconstruct the unknown distribution densities of random transportation costs is absent, a minimax solution to the problem is proposed. In this case, using continuous linear programming, the worst distribution densities of costs are determined, and in this worst case scenario, the best plan is found. The developed procedure for solving a transport problem under conditions of uncertainty in the initial data contains, as the main element of the computational scheme, a method for the exact solution of the problem of fractional nonlinear programming. This method has an independent value, adding to the arsenal of known nonlinear optimization methods. Finally, it should be noted that the proposed method, which solves the problem of optimizing transportation for random input data, can be generalized to the case when these data are described in terms of the theory of fuzzy sets.

The directions of further research are related to the development of methods for solving the considered problems for cases when the uncertainty of the initial data is described in terms of fuzzy [16-18] or imprecise [19] mathematics. Possible solutions can be obtained using the methods proposed in [20-22], where it is theoretically and experimentally shown how to do this using the apparatus of fuzzy mathematics for applied problems of process control under conditions of uncertainty. Based on this, it is possible to talk about the practical usefulness of the results obtained, determined by the possibility of their direct use in solving real problems.

\section{Conclusions}

1. The problem of forming a transportation plan are considered in the «supplier - consumers» system in conditions when the values of the cost of transportation are random values. The urgency of this problem is an inevitable consequence of the real uncertainty in the description of the initial data of practical problems. An exact method is proposed for solving this problem according to the criterion - the probability that the random total cost of implementing the plan will exceed the threshold, acceptable value. The solution is achieved using an iterative procedure, at each step of which a quadratic programming problem is solved. In order to speed up this procedure, an alternative method is proposed based on a single solution of the fractional-quadratic programming problem.

2. A method for solving the problem of optimization of transportation for a situation when the density of distribution of a random cost cannot be correctly determined is considered. In this case, the solution to the problem was obtained under the assumption of the worst distribution density of the random cost of transportation. The solution is implemented using the theory of continuous linear programming.

\section{References}

[1] Birman, I. Ya. (1962). Transportnaya zadacha lineynogo programmirovaniya. Moscow: Izd. ek. lit., 262.

[2] Nesterov, E. P. (1962). Transportnaya zadacha lineynogo programmirovaniya. Moscow: Izd. MPS, 189.

[3] Yudin, D. B., Gol'shteyn, E. G. (1969). Zadachi lineynogo programmirovaniya transportnogo tipa. Moscow: «Sov. radio», 382.

[4] Chumakov, V. B. (2006). Reshenie zadach organizatsii avtomobil'nyh perevozok v usloviyah neopredelennosti sostoyaniya regional'nyh transportnyh sistem. Vestnik Severo-Kavkazskogo federal'nogo universiteta, 5, 22-26.

[5] Malakhov, V. P., Kondratenko, H. V. (2002). Imitatsiyni modeli ta alhorytmy formuvannia marshrutiv i traiektoriy na osnovi nechitkoi lohiky. Trudy Odesskogo politehnicheskogo universiteta, 2, 72-80.

[6] Rosandich, R. G. (2005). Quantification on Uncertainty in Transportation Infrastructure Projects. Santiago: Economics planning.

[7] Borisova, E. A., Finaev, V. I. (2006). Triaksial'naya raspredelitel'naya zadacha s nechetkimi parametrami. Izvestiya Yuzhnogo federal'nogo universiteta. Tehnicheskie nauki, 8, 17-21.

[8] Borisova, E. A., Finaev, V. I. (2007). Trehindeksnye raspredelitel'nye zadachi s nechetkimi parametrami. Taganrog: TTI YUFU, 190.

[9] Ermol'ev, Yu. M. (1976). Metody stohasticheskogo programmirovaniya. Moscow: Nauka, 239.

[10] Kaplinskii, A. I., Poznyak, A. S., Propoy, A. I. (1971). On certain methods of solving problems of stochastic programming. Automation and Remote Control, 32 (10), 1609-1616.

[11] Porotskiy, S. M. (1977). Stohasticheskie zadachi transportnogo tipa. Izv. AN SSSR. Tehn. Kibernetika, 6, 34-39.

[12] Yudin, D. B., Yudin, A. D. (2009). Ekstremal'nye modeli v ekonomike. Moscow: LIBROKOM, 312.

[13] Yudin, D. B. (1979). Zadachi i metody stohasticheskogo programmirovaniya. Moscow: «Sov. radio», 385.

[14] Seraya, O. V. (2010). Mnogomernye modeli logistiki v usloviyah neopredelennosti. Kharkiv: VOP Stetsenko, 512. 
[15] Raskin, L. G., Kirichenko, I. O. (2005). Kontinual'noe lineynoe programmirovanie. Kharkiv: VIVV, 175.

[16] Zadeh, L. A. (1965). Fuzzy sets. Information and Control, 8 (3), 338-353. doi: https://doi.org/10.1016/s0019-9958(65)90241-x

[17] Dyubua, D., Prad, A. (1990). Teoriya vozmozhnostey. Prilozhenie k predstavleniyu znaniy v informatike. Moscow: Radio i svyaz', 286.

[18] Raskin, L. G., Seraya, O. V. (2008). Nechetkaya matematika. Osnovy teorii. Prilozheniya. Kharkiv: Parus, 352.

[19] Pawlak, Z. (1982). Rough sets. International Journal of Computer \& Information Sciences, 11 (5), 341-356. doi: https:// doi.org/10.1007/bf01001956

[20] Domin, D. (2013). Artificial orthogonalization in searching of optimal control of technological processes under uncertainty conditions. Eastern-European Journal of Enterprise Technologies, 5 (9 (65)), 45-53. doi: https://doi.org/10.15587/1729-4061.2013.18452

[21] Raskin, L., Sira, O. (2016). Fuzzy models of rough mathematics. Eastern-European Journal of Enterprise Technologies, 6 (4 (84)), 53-60. doi: https://doi.org/10.15587/1729-4061.2016.86739

[22] Raskin, L., Sira, O. (2016). Method of solving fuzzy problems of mathematical programming. Eastern-European Journal of Enterprise Technologies, 5 (4 (83)), 23-28. doi: https://doi.org/10.15587/1729-4061.2016.81292

Received date 29.10.2020

(C) The Author(s) 2021

Accepted date 12.02.2021

Published date 31.03.2021

This is an open access article under the CC BY license (http://creativecommons.org/licenses/by/4.0).

How to cite: Raskin, L., Sira, O., Parfeniuk, Y., Bazilevych, K. (2021). Development of methods for supply management in transportation networks under conditions of uncertainty of transportation cost values. EUREKA: Physics and Engineering, 2, $108-123$. doi: https://doi.org/10.21303/2461-4262.2021.001691 